\title{
On the orbital and physical parameters of the HDE 226868/Cygnus X-1 binary system
}

\author{
Lorenzo Iorio $^{1}$
}

(C) Springer-Verlag $\bullet \bullet \bullet$

\begin{abstract}
In this paper we explore the consequences of the recent determination of the mass $m=(8.7 \pm 0.8) M_{\odot}$ of Cygnus X-1, obtained from the Quasi-Periodic Oscillation (QPO)-photon index correlation scaling, on the orbital and physical properties of the binary system HDE 226868/Cygnus X-1. By using such a result and the latest spectroscopic optical data of the HDE 226868 supergiant star we get $M=(24 \pm 5) M_{\odot}$ for its mass. It turns out that deviations from the third Kepler law significant at more than 1-sigma level would occur if the inclination $i$ of the system's orbital plane to the plane of the sky falls outside the range $\approx 41 \div 56 \mathrm{deg}$ : such deviations cannot be due to the first post-Newtonian (1PN) correction to the orbital period because of its smallness; interpreted in the framework of the Newtonian theory of gravitation as due to the stellar quadrupole mass moment $Q$, they are unphysical because $Q$ would take unreasonably large values. By conservatively assuming that the third Kepler law is an adequate model for the orbital period we obtain $i=(48 \pm 7) \mathrm{deg}$ which yields for the relative semimajor axis $a=(42 \pm 9) R_{\odot}(\approx 0.2$ $\mathrm{AU})$.
\end{abstract}

Keywords black holes: individual (Cyg X-1)-stars: individual (HDE 226868)-X-rays: binaries-X-rays: individual (Cyg X-1)

\section{Introduction}

Cygnus $\mathrm{X}-1$, one of the brightest high-energy sources in the sky, with an average $1-200 \mathrm{keV}$ energy flux of $\sim 3 \times 10^{-8} \mathrm{erg} \mathrm{cm}^{-2} \mathrm{~s}^{-1}$, was discovered by Giacconi et al. (1967) and soon became one of the most

Lorenzo Iorio

INFN-Sezione di Pisa. Permanent address for correspondence: Viale Unità di Italia 68, 70125, Bari (BA), Italy. intensively studied X-ray sources. After the detection of its rapid X-ray variability (Oda et al. 1971) and the identification of its optical counterpart with the O9.7 Iab supergiant star HDE 226868 (Bolton 1972; Webster \& Murdin 1972), it was considered as one of the most likely black hole candidates. The X-ray emission in Cygnus X-1 is powered mainly by accretion from the strong stellar wind from HDE 226868 (Petterson 1978).

In this paper we investigate some features of the orbital geometry of the HDE 226868/Cygnus X-1 binary system in order to check the compatibility of the so far obtained spectroscopic data of HDE 226868 (Gies et al. 2003) with the tight constraints on the black hole mass $m=(8.7 \pm 0.8) M_{\odot}$ recently determined by Shaposhnikov \& Titarchuk (2007) who exploited the existing correlation (Shaposhnikov \& Titarchuk 2006) between the low frequency Quasi-Periodic Oscillations (LF QPO) and the photon index of the power law spectral component of the Cygnus X-1 X-ray emission. We will look for deviations from the third Kepler law, which will not be assumed a priori valid, by discussing the conditions at which they occur and their physical plausibility.

\section{Deviations from the third Kepler law}

The analysis of 115 optical spectra of the $\mathrm{H} \alpha$ emission line of HDE 226868 for the 1998-2002 interval allowed Gies et al. (2003) to phenomenologically determine the orbital period $P$ and the projected velocity semiamplitude $K$ (see Table1): for $P$ the same value, within the errors, of the one obtained by Brocksopp et al. (1999), who used a 26-yr data set, was obtained. A previous orbital fit can be found, e.g., in (Gies et al. 1982).

From $P$ and $K$, in turn, it is possible to obtain the stellar projected barycentric semimajor axis $x_{M} \equiv$ 
Table 1 Relevant orbital parameters of the HDE 226868/Cygnus X-1 binary system. $P$ is the orbital period (Brocksopp et al. 1999), $K$ is the projected velocity semiamplitude (Gies et al. 2003), $q \equiv m / M$ is the ratio of the black hole to the star mass (Gies et al. 2003), $m$ is the black hole's mass (Shaposhnikov \& Titarchuk 2007). All of them have been determined independently of the third Kepler law: $P, K, q$ from optical spectroscopy of HDE 226868, and $m$ from the analysis of certain properties of the Cygnus $\mathrm{X}-1$ X-ray emission.

\begin{tabular}{llll}
\hline$P(\mathrm{~d})$ & $K\left(\mathrm{~km} \mathrm{~s}^{-1}\right)$ & $q$ & $m\left(M_{\odot}\right)$ \\
\hline $5.599829(16)$ & $75.6(7)$ & $0.36(5)$ & $8.7(8)$ \\
\hline
\end{tabular}

$a_{M} \sin i$, where $i$ is the inclination angle of the orbital plane to the plane of the sky, as

$x_{M}=\frac{P K}{2 \pi}=(8.37 \pm 0.07) R_{\odot}$.

An important result of the spectroscopical analysis by Gies et al. (2003) is that the emission component of the radial velocity is compatible with a mass ratio

$q \equiv \frac{m}{M}=0.36 \pm 0.05$

The recent result by Shaposhnikov \& Titarchuk (2007) for $m$ yields for the mass of HDE 226868

$M=(24 \pm 5) M_{\odot}$

it disagrees with $M=17.8 M_{\odot}$ by Herrero et al. (1995) which, among other things, relies upon various estimates of $i$. Even larger is the disagreement with $M=(40 \pm 5) M_{\odot}$ obtained by Ziółkowski (2005) with evolutionary calculation for the most likely intervals of the values of the distance and of the effective temperature1: 1.95 to $2.35 \mathrm{kpc}$ and 30000 to $31000 \mathrm{~K}$. It is important to note that the method by Shaposhnikov \& Titarchuk (2007) is not only independent of the lingering uncertainty in the system's distance from us, but it was also robustly tested by reproducing the masses of other black hole candidates, like the one in the microquasar GRS $1915+$ 105 (Castro-Tirado et al. 1992), obtained with IR (Greiner et al. 2001) and X-ray Shrader \& Titarchuk 2003) observations. Thus, in the following we will trustworthy rely upon such a result. Note that the mass estimate by Shaposhnikov \& Titarchuk (2007) for $m$ is well within the range $4.8 M_{\odot}<m<14.7 M_{\odot}$ obtained by Herrero et al. (1995).

${ }^{1}$ Extending the intervals of these parameters to 1.8 to $2.35 \mathrm{kpc}$ and 28000 to $32000 \mathrm{~K}$, Ziółkowski (2005) obtained a range $29 \div 50$ for $M$.
We are now in the position of looking for genuine deviations from the third Kepler law by comparing the phenomenologically determined period $P$ to the calculated Keplerian one which, from eq. (11) and

$a=\left(1+\frac{1}{q}\right) \frac{x_{M}}{\sin i}$

for the relative semimajor axis, can be expressed as

$P^{\mathrm{Kep}}=\left(\frac{1+q}{q}\right) \sqrt{\frac{1}{2 \pi G m}\left(\frac{K P}{\sin i}\right)^{3}}$

in terms of quantities determined independently of the third Kepler law itself: note that eq. (5) does not depend on $M$. The overall uncertainty $\delta P^{\text {kep }}$ in eq. (5), which will be considered as a function of $i$, can be conservatively evaluated by linearly adding the various mismodelled terms due to $\delta q, \delta G, \delta m, \delta K, \delta P$ as

$$
\begin{aligned}
& \left.\delta P^{\mathrm{Kep}}\right|_{q} \leq \frac{P^{\mathrm{Kep}}}{(1+q)}\left(\frac{\delta q}{q}\right), \\
& \left.\delta P^{\mathrm{Kep}}\right|_{G} \leq \frac{P^{\mathrm{Kep}}}{2}\left(\frac{\delta G}{G}\right), \\
& \left.\delta P^{\mathrm{Kep}}\right|_{m} \leq \frac{P^{\mathrm{Kep}}}{2}\left(\frac{\delta m}{m}\right), \\
& \left.\delta P^{\mathrm{Kep}}\right|_{K} \leq\left(\frac{3}{2}\right) P^{\mathrm{Kep}}\left(\frac{\delta K}{K}\right), \\
& \left.\delta P^{\mathrm{Kep}}\right|_{P} \leq\left(\frac{3}{2}\right) P^{\mathrm{Kep}}\left(\frac{\delta P}{P}\right) .
\end{aligned}
$$

In Figure 1 we plot the ratio

$$
\frac{\Delta P}{\delta(\Delta P)} \equiv \frac{\left|P-P^{\mathrm{Kep}}\right|}{\delta P+\delta P^{\mathrm{Kep}}}
$$

as a function of the inclination; outside the range 41 $\operatorname{deg} \lesssim i \lesssim 56 \mathrm{deg}$, in which the purely Keplerian period is an adequate model for the measured orbital one, deviations from the third Kepler law significant at more than 1-sigma level would occur; in particular, the estimate $i=(30 \pm 7)$ deg by Gies et al. (2003) would yield a discrepancy significant at 4/2-sigma-level, while for $i=35 \mathrm{deg}$, chosen by Herrero et al. (1995) because lying in the middle of almost all determinations in literature, the deviation from the third Kepler law would amount to 2-sigma. Can such deviations be considered physically meaningful?

In principle, also the first post-Newtonian (1PN) correction to the Keplerian period (Damour \& Deruelle 1986),

$P^{1 \mathrm{PN}}=\frac{3}{\sqrt{2} c^{2}}\left(\frac{1+q}{q}\right) \sqrt{\frac{\pi G m K P}{\sin i}}\left[1-\frac{q}{3(1+q)^{2}}\right]$ 


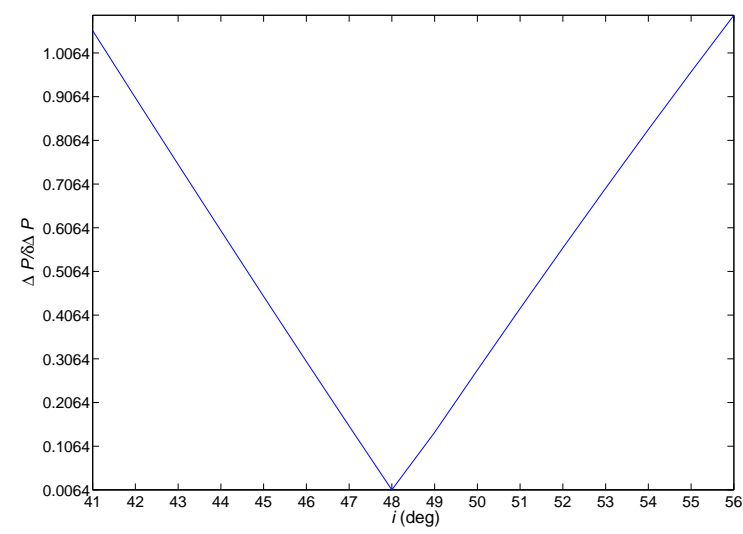

Fig. 1 Discrepancy between the phenomenologically measured orbital period $P$ and the computed Keplerian one $P^{\mathrm{Kep}}$ as function of the inclination $i$.

here expressed in terms of the phenomenologically determined quantities, should be taken into account in modeling the orbital period; however, it is not possible that eq. (8) is the cause of the significant discrepancies because it is six orders of magnitude smaller than $\delta P^{\mathrm{Kep}}$ itself for all values of $i$. Note that considering the possibility that $1 \mathrm{PN}$ terms may have some observable effects in so close binary systems should not be considered trivial since for, e.g., the exoplanet HD209458b the accuracy in determining its 3.5 d orbital period would, in fact, allow to detect 2 the relativistic correction Iorio 2006).

Let us see what happens if we add to the Keplerian period the correction due to the quadrupole mass moment $Q$ of the system: in terms of the determined parameters we have, from eq. (23),

$Q=\frac{\kappa^{2}}{3 \sqrt{2}} \sqrt{\left(\frac{m P}{\pi}\right)^{3} \frac{G K}{\sin i}}-\frac{m \kappa^{3}}{6}\left(\frac{K P}{\pi \sin i}\right)^{2}$,

where we have posed

$\kappa \equiv \frac{1+q}{q}$.

${ }^{2}$ The uncertainties in the system's parameters induce systematic errors which prevent from implementing such a goal.
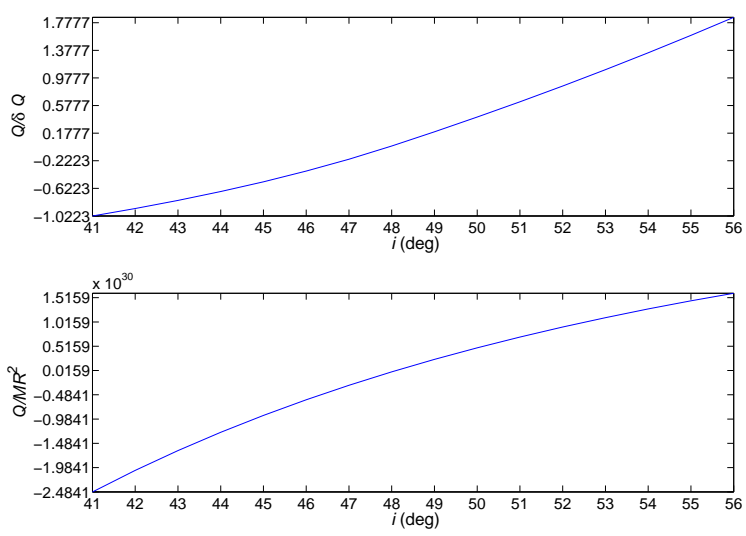

Fig. 2 Upper panel: $Q / \delta Q$. Lower panel: $Q / M R^{2}$ as functions of the inclination $i$.

The total uncertainty in $Q$ can be conservatively evaluated by summing the various contributions

$$
\begin{aligned}
& \left.\delta Q\right|_{G} \leq\left|\frac{\kappa^{2}}{6 \sqrt{2}} \sqrt{\left(\frac{m P}{\pi}\right)^{3} \frac{K}{G \sin i}}\right| \delta G, \\
& \left.\delta Q\right|_{m} \leq\left|\frac{\kappa^{2}}{2 \sqrt{2}} \sqrt{\left(\frac{P}{\pi}\right)^{3} \frac{G m K}{\sin i}}-\frac{\kappa^{3}}{6}\left(\frac{K P}{\pi \sin i}\right)^{2}\right| \delta m, \\
& \left.\delta Q\right|_{P} \leq\left|\frac{\kappa^{2}}{2 \sqrt{2}} \sqrt{\left(\frac{m}{\pi}\right)^{3} \frac{G P K}{\sin i}}-\frac{m P \kappa^{3}}{3}\left(\frac{K}{\pi \sin i}\right)^{2}\right| \delta P, \\
& \left.\delta Q\right|_{K} \leq\left|\frac{\kappa^{2}}{6 \sqrt{2}} \sqrt{\left(\frac{m P}{\pi}\right)^{3} \frac{G}{K \sin i}}-\frac{m K \kappa^{3}}{3}\left(\frac{P}{\pi \sin i}\right)^{2}\right| \delta K, \\
& \left.\delta Q\right|_{q} \leq\left|\frac{\sqrt{2}}{3} \frac{\kappa}{q^{2}} \sqrt{\left(\frac{m P}{\pi}\right)^{3} \frac{G K}{\sin i}}-\frac{m}{2}\left(\frac{\kappa}{q}\right)^{2}\left(\frac{K P}{\pi \sin i}\right)^{2}\right| \delta q .
\end{aligned}
$$

In Figure 2 we plot $|Q| / \delta Q$ (upper panel) and $3 / M R^{2}$ (lower panel): we see that ascribing the deviation from the third Kepler law, when it becomes significant, to the HDE 226868's quadrupole mass moment leads to meaningless results since $Q$ would assume values incompatible with zero at more than 1-sigma level, but unreasonably large.

By conservatively assuming that no deviations from the third Kepler law occur, given the present-day level of accuracy in knowing the system's parameters, it is possible to determine $i$ as

$i=\arcsin \left[K P^{1 / 3}\left(2 \pi G m \kappa^{-2}\right)^{-1 / 3}\right]=(48 \pm 7) \mathrm{deg}$.

${ }^{3}$ We used $R=17 R_{\odot} \quad$ Herrero et al. 1995); according to Ziółkowski (2005), $R / R_{\odot}=10.59 d$, with $d$ in kpc: $d \approx 1.8-2.35$ kpc. 


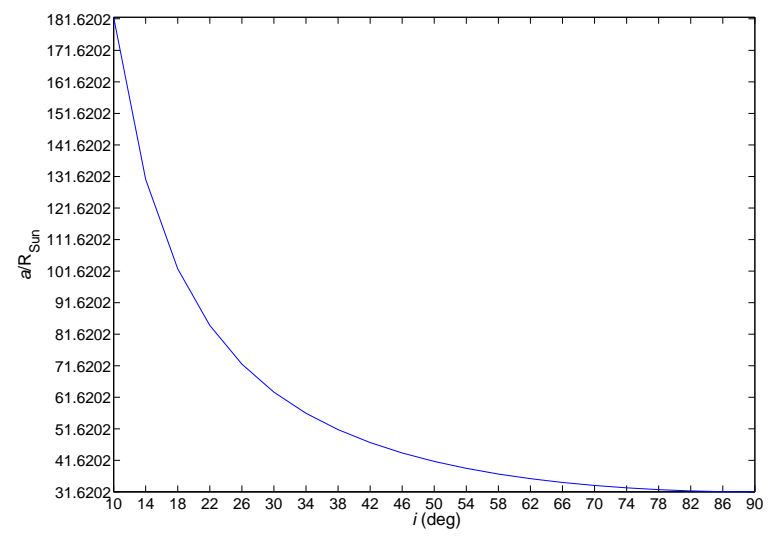

Fig. 3 Relative semimajor axis $a$, in Solar radii, as function of the inclination $i$.

In regard to the inclination $i$, Gies et al. (2003) obtain

$i=(30 \pm 7) \operatorname{deg}$

from the measured emission and absorption radial velocities and by making certain assumptions about isotropy of the outflow velocity; after noting that their result is compatible with other estimates 4 like $28 \mathrm{deg}$ $\leq i \leq 38 \mathrm{deg}$ from light curve analysis by Gies et al. (1986a) and $10 \mathrm{deg} \leq i \leq 40 \mathrm{deg}$ from fitting the X-ray emission curve by Wen et al. (1999), Gies et al. (2003) conclude that eq. (13) is likely a lower limit. Our result, which is incompatible with the one by Gies et al. (2003), being, instead, compatible with, e.g., the result by Dolan \& Tapia (1989), can be useful in terms of anisotropy in the wind outflow velocity (Friend \& Castor 1982).

It is also interesting to evaluate the relative separation of HDE 226868 and Cygnus X-1 (see Figure 31): such an information may be helpful about HDE 226868's dimensions. The relative semimajor axis, according to eq. (11) and eq. (41) which are independent of the third Kepler law and on the mass $m$ of Cygnus X-1, amounts to $a=(42 \pm 9) R_{\odot}(\approx 0.2 \mathrm{AU})$ for $i=48 \mathrm{deg}$; the minimum value, i.e. $a=31.6 R_{\odot}(0.1 \mathrm{AU})$, would occur for the edge-on configuration while for $i=10 \mathrm{deg}$ it amounts to about 181 Solar radii (0.8 AU).

Note that, should one consider $M=(40 \pm 5) M_{\odot}$ inferred by Ziółkowski (2005), the black hole's mass by Shaposhnikov \& Titarchuk (2007) would yield $q=$

${ }^{4}$ Optical polarimetry of HDE 226868 yields $25 \mathrm{deg} \leq i \leq 67 \mathrm{deg}$ (Dolan \& Tapia 1989) and $20 \mathrm{deg} \leq i \leq 40 \mathrm{deg}$ (Daniel 1981).

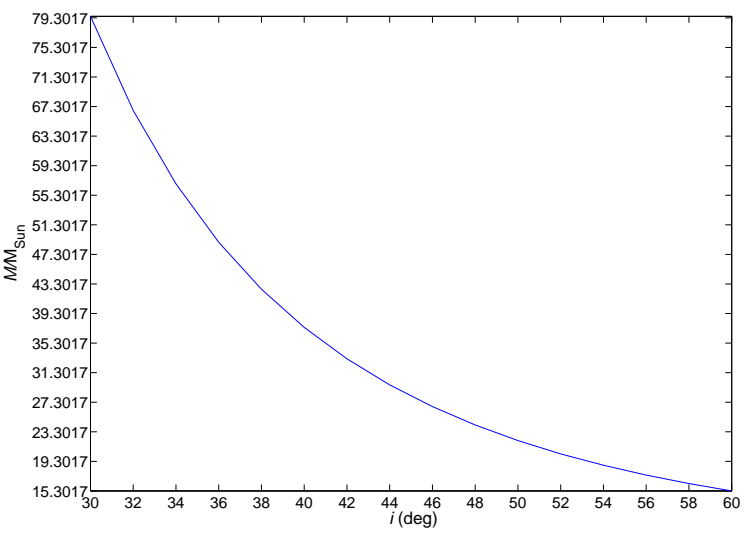

Fig. 4 Mass of HDE 226868, in Solar masses, as function of the inclination $i$. The third Kepler law was used.

$0.22 \pm 0.05$. Such a value, which is inconsistent at 1.4sigma level with the one by Gies et al. (2003), would lead to unreasonably large values for the quadrupole mass moment for $i \lesssim 45 \mathrm{deg}$, being all the other values up to 90 deg compatible with zero $Q$ and no deviations from the third Kepler law. Near edge-on orbital configurations imply that eclipses in the X-ray emission of Cygnus X-1 would be allowed ( $i \gtrsim 65 \mathrm{deg}$ ), but they have never been detected. Thus, the estimate for the HDE 226868's mass by Ziółkowski (2005) does not seem plausible. Another line of reasoning is the following. From the third Kepler law we can express the HDE 226868's mass in terms of the spectroscopically determined parameters as

$G M=\left(\frac{P}{2 \pi}\right)(1+q)^{2}\left(\frac{K}{q \sin i}\right)^{3} ;$

as can be noted from Figure $4 M$ assumes the values by Ziółkowski (2005), who relies on the third Kepler law, for $37 \mathrm{deg} \lesssim i \lesssim 41 \mathrm{deg}$; but our previous analysis, independent of $M$ itself, has shown that for such values significant deviations from the third Kepler law would occur (see Figure 1).

Note that our results are compatible with a system distance of about $1.80 \mathrm{kpc}$ (Malvsheva 1997) and an effective temperature $T_{e}=32000 \mathrm{~K}$ (Herrero et al. 1995) of HDE 226868, according to Table 1 of (Ziółkowski 2005) based on the quite general approach by Paczýnski (1974).

\section{Discussion and conclusions}

In this paper we refined some orbital and physical parameters of the HDE 226868/Cygnus X-1 binary system by exploiting the latest spectroscopic optical data 
from HDE 226868 and the recent determination of the mass $m$ of Cygnus X-1 via QPO-index correlation scaling. For the mass of the supergiant star we obtained $M=(24 \pm 5) M_{\odot}$, which is in disagreement with other estimates present in literature. Then, we constrained the inclination $i$ by looking for statistically significant deviations from the third Kepler law which would occur, at more than 1-sigma level, for $i \lesssim 41 \mathrm{deg}$ and $i \gtrsim 56 \mathrm{deg}$. After noting that they cannot be due to the $1 \mathrm{PN}$ correction to the Keplerian period, we concluded that, if attributed to the star's quadrupole mass moment $Q$, they are to be considered unphysical because of the too large values which $Q$ would take in this case. The inspection of the possible values of $Q$ allowed also to consider unlikely the value $M=(40 \pm 5) M_{\odot}$ for $\mathrm{HDE}$ 226868 recently inferred from evolutionary calculation because it would allow for high values of $i$ compatible with eclipses which, instead, are so far absent. Thus, by reasonably assuming that the Keplerian period is an adequate model of the orbital period we determined $i=(48 \pm 7) \mathrm{deg}$. In this case, the relative semimajor axis amounts to $a=(42 \pm 9) R_{\odot}(\approx 0.2 \mathrm{AU})$.

\section{Appendix: the correction to the orbital period due to the quadrupole mass moment}

One of the six Keplerian orbital elements in terms of which it is possible to parameterize the orbital motion in a binary system is the mean anomaly $\mathcal{M}$ defined as $\mathcal{M} \equiv n\left(t-T_{0}\right)$, where $n$ is the mean motion and $T_{0}$ is the time of pericenter passage. The mean motion $n \equiv 2 \pi / P_{\mathrm{b}}$ is inversely proportional to the time elapsed between two consecutive crossings of the pericenter, i.e. the anomalistic period $P_{\mathrm{b}}$. In Newtonian mechanics, for two point-like bodies, $n$ reduces to the usual Keplerian expression $n^{\text {Kep }}=\sqrt{G \mathrm{M} / a^{3}}$, where $a$ is the semi-major axis of the relative orbit and $\mathrm{M} \equiv m_{1}+m_{2}$ is the sum of the masses. In many binary systems the period $P_{\mathrm{b}}$ is accurately determined in a phenomenological, model-independent way, so that it accounts for all the dynamical features of the system, not only those coming from the Newtonian point-like terms, within the measurement precision.

Here we wish to calculate the contribution of the quadrupole mass moment $Q$ to the orbital period in a general way.

By assuming that the spins and the orbital angular momentum are aligned, as done in (Herrero et al. 1995) for the HDE 226868/Cygnus X-1 system, the radial component of the acceleration induced by $Q$ can be cast into the form

$A_{Q}=\frac{3}{2} \frac{G Q}{r^{4}}$ while the other component, i.e. the latitudinal one, vanishes. $A_{Q}$ is small with respect to the usual Newtonian monopole, so that it can be treated perturbatively. In order to derive its impact on the orbital period $P_{\mathrm{b}}$, let us consider the Gauss equation for the variation of the mean anomaly in the case of an entirely radial disturbing acceleration $A$

$\frac{d \mathcal{M}}{d t}=n-\frac{2}{n a} A\left(\frac{r}{a}\right)+\frac{\left(1-e^{2}\right)}{n a e} A \cos f$,

where $f$ is the true anomaly, reckoned from the pericenter. After inserting $A_{Q}$ into the right-hand-side of eq. (16), it must be evaluated onto the unperturbed Keplerian ellipse

$r=\frac{a\left(1-e^{2}\right)}{1+e \cos f}$.

By using (Rov 2005)

$\frac{d f}{d \mathcal{M}}=\left(\frac{a}{r}\right)^{2} \sqrt{1-e^{2}}$

eq. (16) yields

$$
\begin{aligned}
\frac{d f}{d t}= & \frac{n(1+e \cos f)^{2}}{\left(1-e^{2}\right)^{3 / 2}}\left\{1+\frac{3 G Q(1+e \cos f)^{3}}{n^{2} a^{5}\left(1-e^{2}\right)^{3}} \times\right. \\
& \left.\times\left[\frac{\cos f(1+e \cos f)}{2 e}-1\right]\right\}
\end{aligned}
$$

The orbital period can be obtained as

$$
\begin{aligned}
P_{\mathrm{b}} \approx & \frac{\left(1-e^{2}\right)^{3 / 2}}{n} \int_{0}^{2 \pi}\left\{1-\frac{3 G Q(1+e \cos f)^{3}}{n^{2} a^{5}\left(1-e^{2}\right)^{3}} \times\right. \\
& \left.\times\left[\frac{\cos f(1+e \cos f)}{2 e}-1\right]\right\} \frac{d f}{(1+e \cos f)^{2}}(20)
\end{aligned}
$$

From eq. (20) it follows

$P_{\mathrm{b}} \equiv P^{\mathrm{Kep}}+P^{Q}$,

with

$\left\{\begin{array}{l}P^{\mathrm{Kep}}=2 \pi \sqrt{\frac{a^{3}}{G \mathrm{M}}}, \\ P^{Q}=\frac{3 \pi Q}{\sqrt{G a \mathrm{M}^{3}\left(1-e^{2}\right)^{3}}} .\end{array}\right.$

Note that $P^{Q}$ in eq. (22) agrees with the expression of the anomalistic period of a satellite orbiting an oblate planet obtained by Capderou (2005): for a direct comparison $Q=-\mathrm{M} R^{2} J_{2}$, where $J_{2}$ is the first even zonal harmonic of the multipolar expansion of the Newtonian part of the gravitational potential of the central body of mass $\mathrm{M}$ and equatorial radius $R$. 
Solving for $Q$, we finally get

$$
Q=\frac{P_{\mathrm{b}} \sqrt{G a \mathrm{M}^{3}\left(1-e^{2}\right)^{3}}}{3 \pi}-\frac{2}{3} \mathrm{M} a^{2}\left(1-e^{2}\right)^{3 / 2} .
$$




\section{References}

Bolton, C.T.: Nature. 235, 271 (1972)

Brocksopp, C., Tarasov, A.E., Lyuty, V.M., Roche, P.: Astron. Astrophys. 343, 861 (1999)

Capderou, M: Satellites orbits and Missions. SpringerVerlag (2005)

Castro-Tirado, A.J., Brandt, S., Lund, S.: IAU Circ. 5590 (1992)

Damour, T., Deruelle, N.: Ann. Inst. H. Poincaré. 44, 263 (1986)

Daniel, J.-Y.: Astron. Astrophys. 94, 121 (1981)

Dolan, J.F., Tapia, S.: Astrophys. J. 344, 830 (1989)

Friend, D.B., Castor J.I.: Astrophys. J. 261, 293 (1982)

Giacconi, R., Gorenstein, P., Gursky, H., Waters, J.R.: Astrophys. J. 148, L119 (1967)

Gies, D.R., Bolton, C.T.: Astrophys. J. 260, 240 (1982)

Gies, D.R., Bolton, C.T.: Astrophys. J. 304, 371 (1986a)

Gies, D.R., Bolton, C.T., Thomson, J.R., Huang, W., McSwain, M.V., Riddle, R.L., Wang, Z., Wiita, P.J., Wingert, D.W., Csák, B., Kiss, L.L.: Astrophys. J. 583, 424 (2003)

Greiner, J., Cuby, J.G., McCaughrean, M.G.: Nature. 414, $522(2001)$

Herrero, A., Kudritzki, R.P., Gabler, R., Vilchez, J.M., Gabler, A.: Astron. Astrophys. 297, 556 (1995)

Iorio, L.: New Astron. 11, 490 (2006)

Malysheva, L.K.: Astron. Lett. 23, 585 (1997)

Oda, M., Gorenstein, P., Gursky, H., Kellogg, E., Schreier, E., Tananbaum, H., Giacconi, R.: Astrophys. J. 166, L1 (1971)

Paczýnski, B.: Astron. Astrophys. 34, 161 (1974)

Petterson, J.A.: Astrophys. J. 224, 625 (1978)

Roy, A.E.: Orbital Motion, 4th edn. Institute of Physics (2005)

Shaposhnikov, N., Titarchuk, L.G.: Astrophys. J. 643, 1098 (2006)

Shaposhnikov, N., Titarchuk, L.G.: Astrophys. J. 663, 445 (2007)

Shrader, C., Titarchuk, L.G.: Astrophys. J. 598, 168 (2003)

Webster, B.L., Murdin, P.: Nature. 235, 37 (1972)

Wen, L., Cui, W., Levine, A.M., Bradt, H.V.: Astrophys. J. 525, 968 (1999)

Ziółkowski, J.: Mon. Not. R. Astron. Soc.. 358, 851 (2005)

This manuscript was prepared with the AAS LATEX macros v5.2. 